\title{
Analysis of Local Binary Patterns and Uniform Local Binary Patterns for Palm Vein Biometric Recognition
}

\author{
Alicia Aglio-Caballero \\ Belén Ríos-Sánchez, Carmen Sánchez-Ávila \\ María José Melcón de Giles
}

\begin{abstract}
Palm vein recognition has emerged as a novelty highly invariant biometric technique that is difficult to forge due to their internal nature. In this work the texture descriptors Local Binary Patterns (LBP) and Uniform Local Binary Patterns (LBPU) are analyzed as feature extraction methods for biometric verification based on palm veins. Their performance and efficiency has been studied through a multivariate analysis including the impact of different wavelengths and the parameters of the texture descriptor number of neighboors and radio. CASIA Multi-Spectral Palmprint Image Database V1.0 has been used for the evaluation of the system.
\end{abstract}

Keywords-Biometrics, LBP, LBPU, Palm Veins, Pattern Recognition, Texture Analysis

\section{INTRODUCTION}

Palm vein recognition has emerged as a novelty biometric technique which presents some remarkable advantages such as their highly invariance or the difficulty to be forged, derived from their location inside the body. Their placement protect them from external factors such as environmental conditions or handicrafts at the same time that increases the complexity of counterfeits. In addition, veins patterns have been proved to be unique, not only those located at traditionally studied body parts like the retina [1] or the hand [2], but also the hole human body vein pattern [3]. Moreover, the trend toward contact-less biometrics of the last years has derived into new capturing devices, which remove hygienic concerns and make more comfortable the capturing process, therefore improving the acceptance from the users. These capturing devices work on the Near Infrared (NIR) light spectrum, which is absorbed by the haemoglobin of the veins showing them almost black in the images and highly contrasted with the rest of the hand. The use of NIR illumination also remove some typical image processing difficulties related to ambient light variations or changing backgrounds. As a result, veins patterns seems to be a robust alternative to conventional biometrics such as fingerprints, iris or face recognition.

Palm vein feature extraction methods can be divided into two main groups: minutiae-based, which segment the hand veins in a first step and then extract the information of singular points [4]; and texture-based, which gathers linebased, appearance-based, texture descriptor-based and codebased approaches [5]. Particularly, texture based approaches have gained attention during the last years. Mirmohamadsadeghi and Drygajlo introduced in 2011 [6] the application of local texture patterns into the study of the veins patterns using the multi-scale definition of Local Binary Patterns and Local Derivative Patterns. Zhou and A. Kumar [7] applied a Hessianphase-based and the neighbourhood matching Radon transform to extract the palm vein features and compared the results against various techniques that were present in the literature. In 2014, Wenxiong Kang et. al [8] showed the performance improvement of the palm vein LBP extraction by looking at the pixel ratio and adding some techniques like the normalized gradient-based maximal principal curvature algorithm or the kmeans method. In 2015 Jayanti Yumah Sari et al. proposed to apply Local Line Binary Patterns (LLBP) [9] to overcome the vein image clarity problem, being able to do a successfully feature extraction even on images where the veins were barely distinguishable. In the last years, palm vein patterns have also fused with other biometric features in multimodal systems designed to overcome the disadvantages of each individual technique by taking advantage of the strengths of each single modality. In this line, some authors combine the information of different traits contained in the hand such as finger, palm and back-hand vein patterns [10], fingerprints, palm veins and palm lines [11], or palm-veins and palmprints [12], [13]. Other authors combine palm-veins with biometric traits placed on other body parts such as face [14], [15].

In this work, two extended texture descriptors: Local Binary Patterns and Uniform Local Binary Patterns have been evaluated and analysed following an evaluation methodology based on the definitions suggested by the ISO/IDE 19795 norm [16], [17] and a publicly available database, the CASIA MultiSpectral Palmprint Image Database V1.0 [23]. This analysis is aimed not only to evaluate the effect of the descriptor parameters and the wavelength used during the image acquisition, but also to provide a basis against to compare future research. 


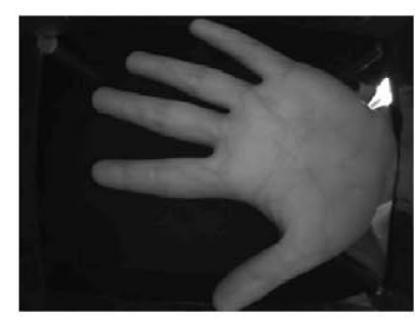

(a) Original Image

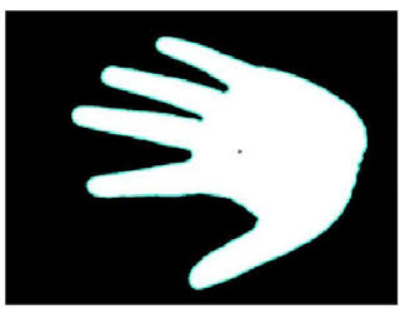

(e) Hand Centroid and Contour

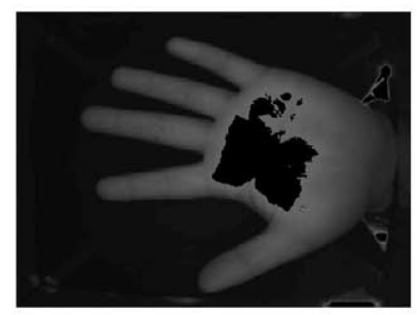

(b) Image smoothing and bright removal

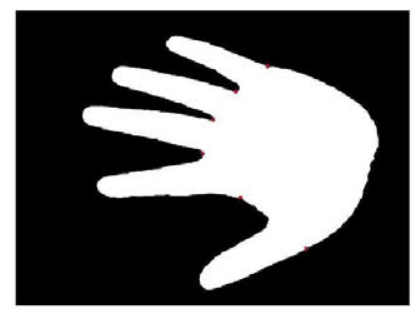

(f) Interfinger Valleys

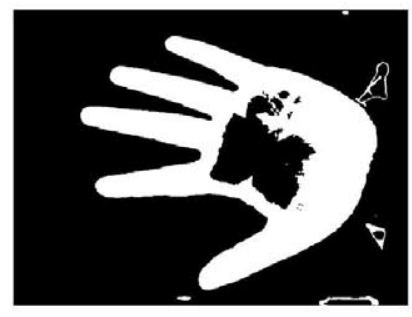

(c) Thresholded Image

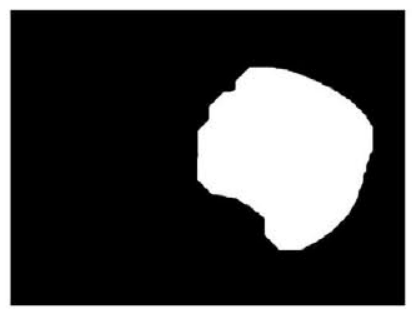

(g) Palm

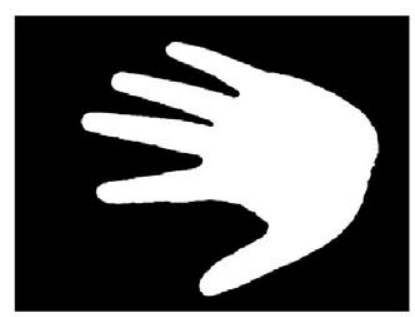

(d) Segmentation Result

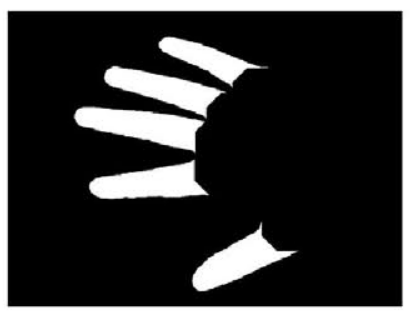

(h) Fingers

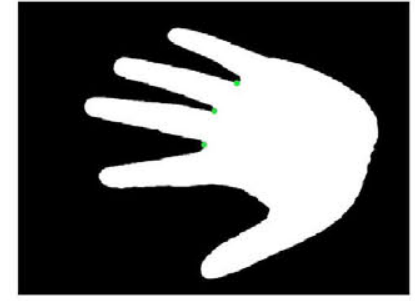

(i) Index-middle, middle-ring and ring-little inter finger valleys

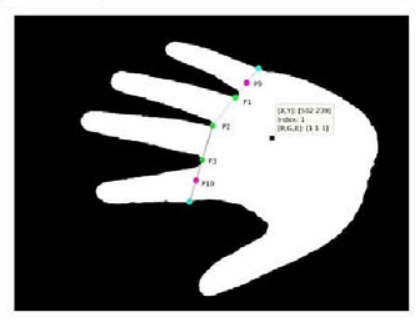

(j) Finger basis points

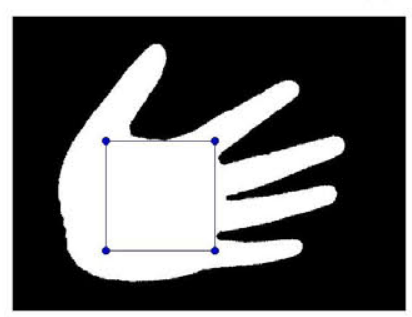

(k) ROI

Fig. 1. Preprocessing

The remaining of this paper is organized as follows. First, feature extraction and image preprocessing are described in Section II. Then, feature comparison approach is detailed in Section III. Next, Section IV presents the evaluation protocol, the database used in the tests and the achieved results. Finally, conclusions are provided in Section V.

\section{FEATURE EXTRACTION}

\section{A. Preprocessing}

To extract reliable and invariant biometric information of the palmveins, it is necessary to locate the central region of the palm, also known as Region of Interest (ROI), which contains most of the palm veins information. To this end, the hand is separated from the background and some meaningful points which allows to extract the ROI are detected. Given the contact-less nature of the images, to obtain more robust features the ROIs are aligned into a common coordinate system to avoid problems related to hand position in different images.

a) Hand Segmentation: First of all, the hand is separated from the background. To this end, the image is smoothed by applying a Gauss filter and spurious bright regions are deleted by removing the higher frequencies. Then, the hand is segmented. Given the high contrast between hand and background provided by the infrared cameras used to capture the hand veins, a simple thresholding approach based on Otsu method [18] has been used to segment the hands. Finally, the result of the segmentation is refined by means of morphological operations of erosion, opening, dilation, closing and dilation conditioned to fill holes. In the case that more than one region remain in the image after de refinement process, the blob with the bigger area is selected as hand. This process is illustrated in Figures 1(a)-1(d).

b) Meaningful Points detection: To calculate the interfinger valleys and other meaningful points which are used to obtain the ROI, the contour and the centroid of the hand are detected in the binary image (Figure 1(e)). Then, euclidean distances between each contour point and the hand centroid are calculated. Those points which are farther from the centroid correspond to fingertips and wrist, while those points between fingertips and wrist extremes which are closer to the centroid coincide with the interfinger valleys and the exterior basis of thumb and little fingers (Figure 1(f)). Next, fingers are isolated by means of morphological operations and identified according to the euclidean distances between their centroids. To this end, fingers are removed from the segmented image by applying an opening operator with disk structural element, giving as a result a new image containing those regions corresponding to the palm and the arm (Figure 1(g)). At that point, the regions which correspond to the fingers are straightforwardly obtained by a simple operation of subtraction (Figure 1(h)). The interfinger valleys between index and middle fingers, middle and ring fingers, and ring and little fingers are identified (Figure 1(i)) as those inter-finger points which are closer to the centroid of the corresponding fingers. 


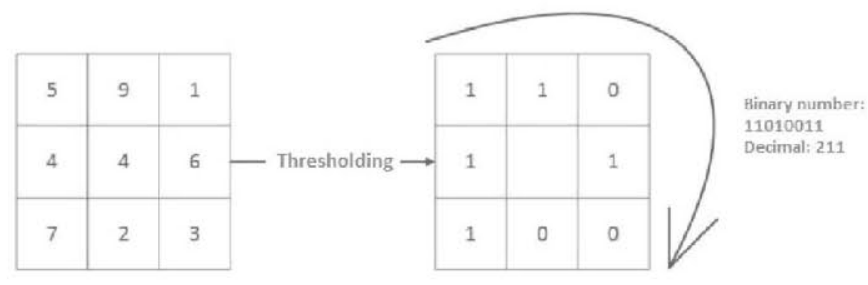

Fig. 2. Original LBP code calculation.

c) ROI detection and Enhancement: Starting from the binary image resulting from the segmentation stage and indexmiddle, middle-ring and ring-little inter-finger valleys ( $P 1-P 3$ in Figure 1(j)), the ROI is detected using a similar approach to that proposed in [19]. Using these points as reference, two vertex $(P 9, P 10$ in Figure $1(\mathrm{j}))$ of the ROI are obtained and aligned in the vertical axe with the thumb finger upwards. Then, taking as a region size the euclidean distance $d$ between $P 9$ and $P 10$, the two segments of size $d$ that are perpendicular to the segment $\overline{P 9 P 10}$, start at $P 9$ and $P 10$, and are closer to the centroid of the hand are detected to complete the ROI (Figure 1(k)). Finally, the obtained ROI is resized to a constant size of $128 \times 128$ pixels and enhanced by means of histogram equalization.

\section{B. Texture Description}

Local Binary Pattern (LBP) has been selected to represent the Palm Veins biometric features. It is a popular texture descriptor introduced in [20] which is characterized by its robustness, simplicity, good performance, and computational efficacy. This operator applies the general definition of texture to a local neighbourhood, representing in a set of binary values (LBP code) the differences between the gray level intensities of a pixel and its neighbours.

First of all, the LBP code is calculated for every pixel in the image employing the intensities corresponding to that pixel and its neighbours. In the original LBP operator a $3 \times 3$ neighbourhood was established. To obtain gray-scale invariance, the intensity of the evaluated pixel (the central pixel) is substracted from the gray value of the neighbours giving as a result a Local Binary Pattern. Then, this pattern is weighted, and the obtained values are added up to obtain the LBP code which contains information about the local features of the texture of the image. This process is illustrated in Figure 2. Finally, the texture of the image is commonly represented by the histogram of the calculated LBP codes.

In 2002, Ojala et al. extended this definition to neighbourhoods of different sizes [21]. The original operator is derived to a general case based on circular symmetry in a region of $\mathrm{P}$ neighbour pixels inside a circle of radius $\mathrm{R}$. Following this principle, the operator is denoted as $L B P_{P, R}$. This way, the LBP operator is defined by two parameters: $\mathrm{P}$ and $\mathrm{R}$, where $P$ represents the number of neighbour pixels and control the angular space quantification, and $\mathrm{R}$ corresponds to the radius of the circle and determines the operator spatial resolution. The operator is defined according to equation 1 :

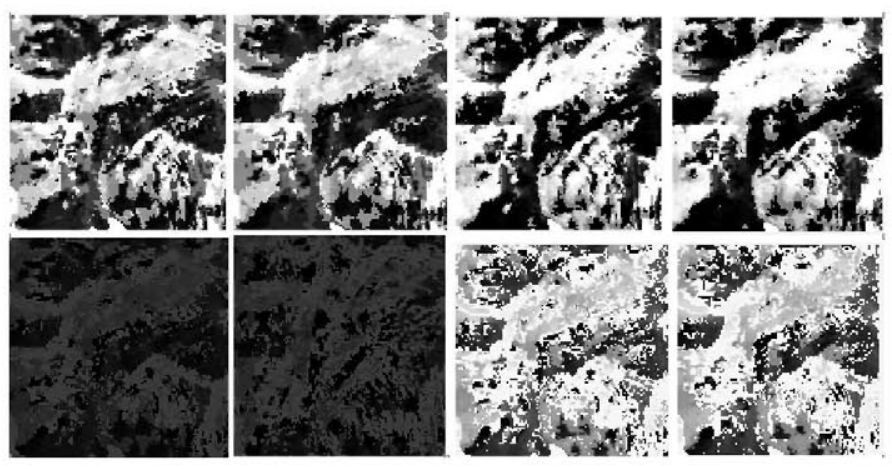

Fig. 3. Comparison of LBP (up) and LBPU (down) using N08R06, N08R08, N16R06, N16R08 (left to right).

$$
L B P_{P, R}=\sum_{p=0}^{p-1} s\left(g_{p}-g_{c}\right) 2^{p}, \quad s(x)=\left\{\begin{array}{l}
1, x \geq 0 \\
0, x<0
\end{array}\right.
$$

In that work Ojala et al. also observed that there are some binary patterns which occur more frequently in the texture description: the Uniform Binary Patterns [21]. These patterns contains very few spatial transitions, there are no more than two bitwise $0 / 1$ changes in the pattern when it is traversed circularly. In this case, to compute the histogram of the image, a different label is given to each uniform binary pattern and another one is assigned to the remaining binary patterns. This way, a shorter texture descriptor is obtained without loosing of relevant information and thus it is sufficiently representative of the distribution of the local features of the image.

Although texture histograms could be directly used as the feature vector corresponding to the user of the biometric system, aimed to add some global information, the image is divided in various regions and a histogram is calculated for each sub-image. The optimum size of these sub-images has been obtained empirically and it is equal to $8 \times 8$ pixels. Finally, histograms were concatenated to compose the final biometric feature vector which describes the palm veins.

Figure 3 depicts the features extracted using LBP and LBPU with different values of radio $(\mathrm{R})$ and neighbourhood (N) parameters.

\section{DiSTANCE-BASED MATCHING}

Distance-based matching has been selected for biometric features comparison due to it is one of the most extended methods given its simplicity and low computational requirements. In particular, the $\chi^{2}$ distance has been used, which is defined in equation 2 . It provides a numeric value as a result, which represents the difference between two feature vectors. Accordingly, the decision policy established in the system is to consider the compared vectors as belonging to the same person if the computed distance is lower than a previously established threshold.

$$
\chi^{2}(S, M)=\sum_{i=1}^{n} \frac{\left(S_{i}-M_{i}\right)^{2}}{S_{i}+M_{i}}
$$


where $S$ and $M$ are the feature vectors to compare and $n$ is the length of these vectors.

Since the enrolment template is composed by more than one feature vectors, the new sample is compared against every feature vector in the template and the lower score is selected.

\section{EVALUATION}

\section{A. Methodology}

A well defined evaluation methodology allows measuring real progress achieved with new research methods as well as pinpointing unsolved problems [22]. For this reason, a technology evaluation protocol based on the definitions suggested by the ISO/IDE 19795 norm [16], [17] and the guidelines provided in [22] has been applied for evaluation purposes.

This evaluation protocol is composed of three parts:

1) Dataset Organization: First of all, it is necessary to separate the original datasets to allow for evaluation tasks. Accordingly, for each database the images of each user are divided into the following datasets:

- Enrolment subset: samples that are used to generate the biometric reference template of each user during the registration into the system.

- Access subset: samples that are employed to simulate the entrance of the users into the system in order to validate it as well as to adjust certain parameters like the acceptance threshold.

2) Computation of Scores: Once the enrolment, and access datasets have been defined, the latter is divided into genuine and impostor samples corresponding to authentic and forger users respectively. Then, the biometric template of each authentic user is created through their enrolment samples. Next, a list of genuine scores is obtained by comparing the access samples against the reference template of the same user, and a list of impostor scores is generated using a zero-effort scheme, where the access samples of a user are compared against the reference templates belonging to the remaining users. Both sets of scores are used to obtain certain metrics that provide information about the performance of the system.

3) Metrics calculation: According to the ISO/IDE 19795 norm - Part1 [16], the performance of the biometric methods has been evaluated through the following metrics:

- Failure-to-enrol rate (FTE): Proportion of the population for whom the system fails to complete the enrolment process.

- Failure-to-acquire rate (FTA): Proportion of verification or identification attempts for which the system fails to capture or locate an image or signal of acceptable quality.

- $\quad$ False non-match rate (FNMR): Proportion of genuine attempts falsely declared not to match the template of the same characteristic from the same user supplying the sample.

- False match rate (FMR): Proportion of zero-effort impostor attempt samples falsely declared to match the compared non-self template.
FTE can be considered as a quality measure about how good is the performance of an algorithm working with a certain sort of images. The False Acceptance Rate and the False Rejection Rate depend on the acceptance threshold of the biometric system that is fixed according to a security policy. A very common policy in biometric systems is to locate the acceptance threshold in the value where FAR and FRR are equal. This value is usually named as Equal Error Rate (EER) and it is an accepted metric to quantify and compare the performance of biometric algorithms.

\section{B. Database description}

The CASIA Multi-Spectral Palmprint Image Database V1.0 [23], collected by The Chinese Academy of Sciences Institute of Automation (CASIA), has been used for evaluation purposes. It contains images of left and right hands belonging to 100 different users. The images were captured using a multiple spectral imaging device which recorded six images at the same time corresponding to six different electromagnetic spectrums: $460 \mathrm{~nm}, 630 \mathrm{~nm}, 700 \mathrm{~nm}, 850 \mathrm{~nm}, 940 \mathrm{~nm}$ and white light. Six images per hand, user and wavelength were acquired. Regarding the capturing conditions, a uniform-colored background was used and the device supplied an evenly distributed illumination. The device had no pegs to restrict postures and positions of palms and captured palm images using a CCD camera fixed on the bottom. Figure 4 shows an example of images belonging to the same user acquired at different wavelengths.

It has been shown that the vascular patterns in left and right hands of the same individual can be distinguished between them due to they present enough dissimilarities [4]. Therefore, in this work the left and right hand of the same person have been considered as belonging to different users, resulting in a total of 200 individuals for the evaluation.

\section{Results}

According to the described evaluation methodology, the set of images of each user has been divided in two subsets: enrolment and evaluation. The three first images of each user have been selected as enrolment samples and the remaining three have been considered as access samples.

A multivariate analysis has been carried out in order to evaluate the influence of different parameters. These parameters and the values involved in this analysis are listed below:

- Pattern: LBP and LBPU.

- Wavelength: $630 \mathrm{~nm}, 700 \mathrm{~nm}, 850 \mathrm{~nm}$ and $940 \mathrm{~nm}$.

- Neighbourhood: 8 and 16.

- $\quad$ Radio: $1,4,6,7$ and 8 .

The obtained results using LBP and LBPU are depicted in Figures 5 and 6 respectively. In addition, the best results achieved by each wavelength and texture descriptor are gathered in Table I. All the results are provided in terms of Equal Error Rate.

From the analysis of the results, the following outcomes can be derived: 


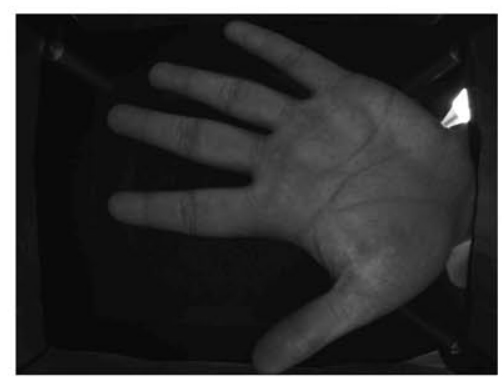

(a) $460 \mathrm{~nm}$

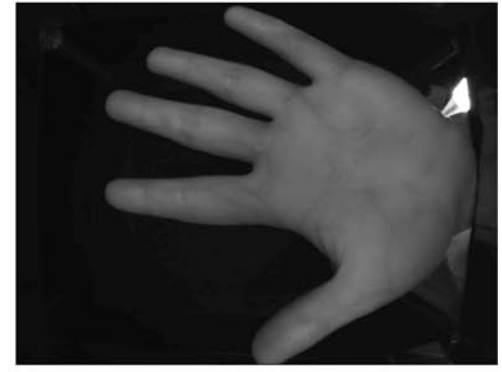

(d) $850 \mathrm{~nm}$

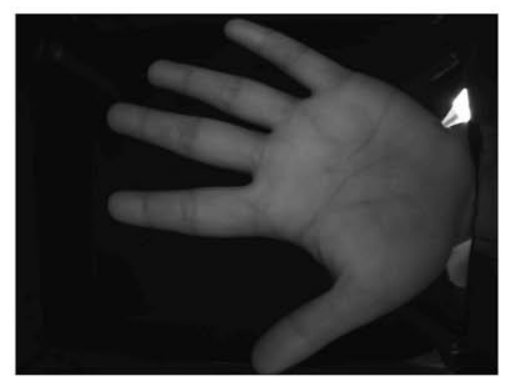

(b) $630 \mathrm{~nm}$

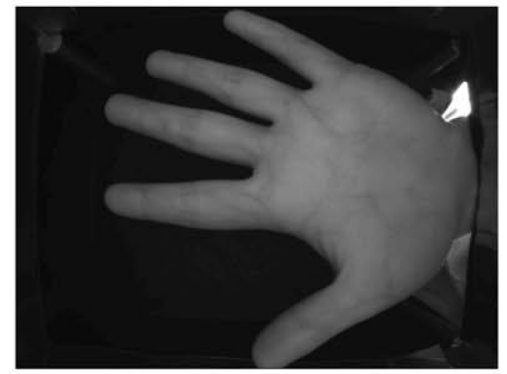

(e) $940 \mathrm{~nm}$

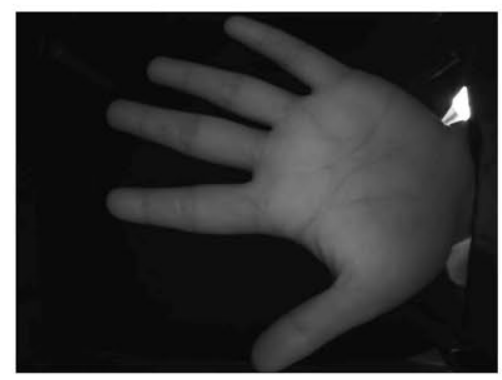

(c) $700 \mathrm{~nm}$

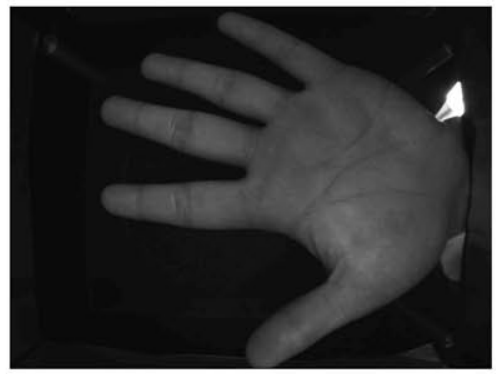

(f) white

Fig. 4. Database sample images.

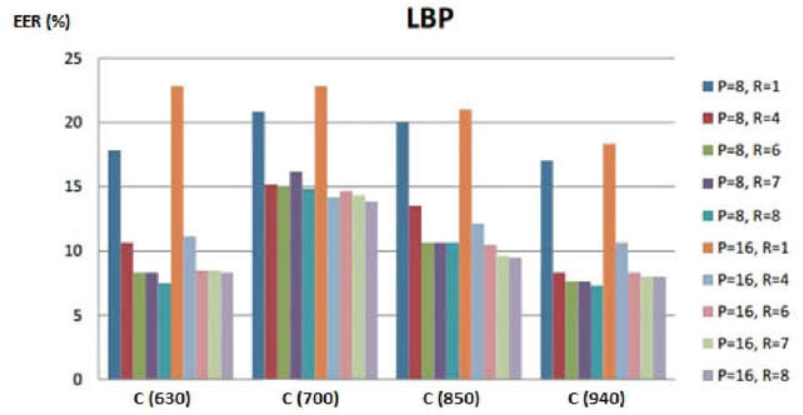

Fig. 5. LBP Tests

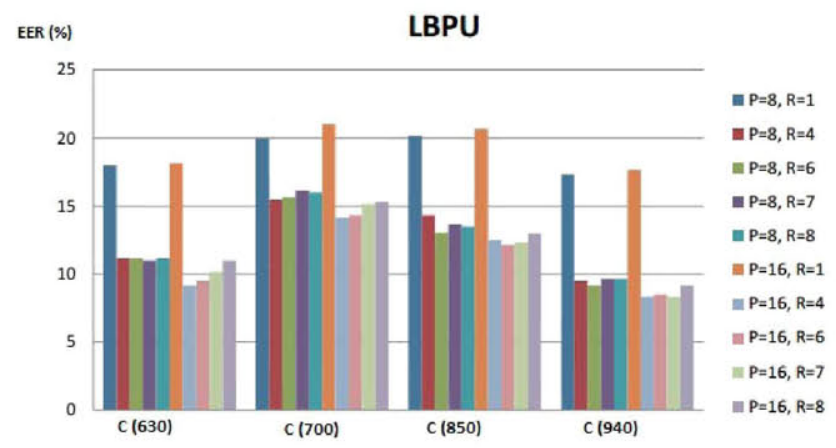

Fig. 6. LBPU Tests

a) Descriptor: As expected, LBP provides slightly better results than LBPU. Nevertheless, the small difference and the computational resources saved by LBPU make it more suitable for real life applications.

\begin{tabular}{cllll}
\hline Wavelength & Descriptor & Neighbours & Radio & EER (\%) \\
630 & LBP & 8 & 8 & 7.56 \\
630 & LBPU & 16 & 6 & 9.16 \\
700 & LBP & 16 & 4 & 14.7 \\
700 & LBPU & 16 & 4 & 14.16 \\
850 & LBP & 16 & 8 & 9.5 \\
850 & LBPU & 16 & 6 & 12.16 \\
940 & LBP & 8 & 8 & 7.33 \\
940 & LBPU & 16 & 4 & 8.33 \\
TABLE I. & BEST RESULTS OBTAINED FOR EACH WAVELENGTH AND \\
\multicolumn{5}{c}{ TEXTURE DESCRIPTOR. }
\end{tabular}

b) Wavelength: The value of the wavelength used to acquire the image clearly influences the system performance. The best results have been obtained at $940 \mathrm{~nm}$, but also good results have been achieved at $630 \mathrm{~nm}$. In this line, a multimodal approach fusing the features extracted at different wavelengths seems to be a good choice to improve the final results. In addition, the good results obtained with the images captured at $630 \mathrm{~nm}$ wavelength, where the palmprint can be seen more clearly, could indicate that a multimodal approach which combines the features contained in the veins patterns and the palmprint could be also a way to improve the system performance.

c) Neighbourhood: The influence of the selected neighbourhood varies between LBP and LBPU. While LBPU always provides better results using 16 neighbours, the accuracy of LBP varies with the number of neighbours and the wavelength.

d) Radio: The selected value for the radio parameter also influences the results. It is evidenced that the performance of the system clearly decreases for small radios. In general, better results are obtained when the radio is equal to 8 in the case of LBP and when radio is equal to 6 or 4 in the case of LBPU. 


\section{Conclusions}

In this work, the texture descriptors Local Binary Patterns (LBP) and Uniform Local Binary Patterns (LBPU) has been analysed as feature extraction methods for contact-less biometric verification based on palm veins. Their performance and efficiency have been studied through a multivariate analysis including the impact of different wavelengths and values for the parameters of the texture descriptor number of neighbours and radio. To this end, a technology evaluation protocol based on the definitions suggested by the ISO/IDE 19795 norm has been followed, and the publicly available CASIA MultiSpectral Palmprint Image Database V1.0 has been used.

\section{ACKNOWLEDGMENT}

This work has been funded by the Spanish Ministry of Economy, Industry and Competitiveness through the project TEC2015-68784-R

\section{REFERENCES}

[1] A. K. Jain, R. Bolle, and S. Pankanti, Eds., Biometrics, Personal Identification in Networked Society: Personal Identification in Networked Society. Norwell, MA, USA: Kluwer Academic Publishers, 1998.

[2] A. Yuksel, L. Akarun, and B. Sankur, "Biometric identification through hand vein patterns," in 2010 International Workshop on Emerging Techniques and Challenges for Hand-Based Biometrics, Aug 2010, pp. $1-6$.

[3] M. Willmore, "Infra-red imaging and pattern recognition system," Sep. 27 1994, uS Patent 5,351,303. [Online]. Available: http: //www.google.com/patents/US5351303

[4] D. Verma and S. Dubey, "A survey on biometric authentication techniques using palm vein feature," Journal of Global Research in Computer Science, vol. 5, no. 8, Aug. 2014.

[5] C. R. Roshni, C. Merin, and M. M. C. M., "Literature survey on contactless palm vein recognition," International Journal of Computer Science Trends and Technology (IJCST), vol. 3, no. 5, pp. 250-255, 2015

[6] L. Mirmohamadsadeghi and A. Drygajlo, "Palm vein recognition with local binary patterns and local derivative patterns," in 2011 International Joint Conference on Biometrics (IJCB), Oct 2011, pp. 1-6.

[7] Y. Zhou and A. Kumar, "Human identification using palm-vein images," IEEE Transactions on Information Forensics and Security, vol. 6, no. 4, pp. 1259-1274, Dec 2011.

[8] W. Kang and Q. Wu, "Contactless palm vein recognition using a mutual foreground-based local binary pattern," IEEE Transactions on Information Forensics and Security, vol. 9, no. 11, pp. 1974-1985, Nov 2014.
[9] J. Y. Sari, C. Fatichah, and N. Suciati, "Local line binary pattern for feature extraction on palm vein recognition," Jurnal ilmu komputer dan informasi, vol, 8, no. 2, 2015.

[10] S. Barathi, R. Sudhakar, and V. E. Balas, Acta Polytechnica Hungarica, vol. 12 , no. 3, 2015

[11] M. Shahin, A. Badawi, and M. Rasmy, "A Multimodal Hand Vein, Hand Geometry, and Fingerprint Prototype Design for High Security Biometrics," in 2008 Cairo International Biomedical Engineering Conference, Dec 2008 , pp. $1-6$.

[12] R. Fuksis, A. Kadikis, and M. Greitans, "Biohashing and Fusion of Palmprint and Palm Vein Biometric Data," in 2011 International Conference on Hand-Based Biometrics, Nov 2011, pp. 1-6.

[13] M. Ong, T. Connie, A. Jin, and A. Jin, "Design and implementation of a contactless palm print and palm vein sensor," in 2010 11th International Conference on Control Automation Robotics Vision, Dec 2010, pp. $1268-1273$.

[14] B. Prasanalakshmi, A. Kannammal, and R. Sridevi, "Multimodal biometric cryptosystem involving face, fingerprint and palm vein," IJCSI International Journal of Computer Science Issues, vol. 8, no. 1, 2011.

[15] S. Bahgat, S. Ghoniemy, and M. Alotaibi, "Proposed multi-modal palm veins-face biometric authentication," International Journal of Advanced Computer Scienceand Applications, vol, 4, no. 6, 2013.

[16] ISO/IEC 19795-1:2007: Information technology - Biometric performance testing and reporting - Part 1: Principles and framework, International Organization for Standardization (ISO), 2007.

[17] ISO/IEC 19795-2:2007: Information technology - Biometric performance testing and reporting - Part 2: Testing methodologies for technology and scenario evaluation, International Organization for Standardization (ISO), 2007.

[18] N. Otsu, "A Threshold Selection Method from Gray-level Histograms," IEEE Transactions on Systems, Man and Cybernetics, vol. 9, no. 1, pp. 62-66, 1979

[19] M. Ong, T. Connie, and A. Jin, "A contactless biometric system using multiple hand features." Journal of Visual Communication and Image Representation, vol. 23, no. 7, pp. 1068 - 1084, 2012.

[20] T. Ojala, M. Pietikäinen, and D. Harwood, "A comparative study of texture measures with classification based on featured distributions," Pattern Recognition, vol. 29, no. 1, pp. 51-59, Jan 1996.

[21] T. Ojala, M. Pietikainen, and T. Maenpaa, "Multiresolution gray-scale and rotation invariant texture classification with local binary patterns," IEEE Transactions on Pattern Analysis and Machine Intelligence, vol. 24, no. 7, pp. 971-987, July 2002.

[22] D. Petrovska-Delacrétaz, G. Chollet, and B. Dorizzi, Eds., Guide to Biometric Reference Systems and Performance Evaluation, 1st ed. Springer-Verlag London, 2009.

[23] “Casia-ms-palmprintv1." [Online]. Available: http://biometrics.idealtest. org/ 